\title{
Development of an Advanced Antineutrino Detector for Reactor Monitoring
}

\author{
T. Classen ${ }^{\mathrm{a}, *}$, A. Bernstein ${ }^{\mathrm{a}}$, N. S. Bowden ${ }^{\mathrm{a}}$, B. Cabrera-Palmer ${ }^{\mathrm{b}}$, A. Ho $^{\mathrm{c}, 1}$, G. Jonkmans $^{\mathrm{c}, 2}$, L. Kogler ${ }^{\mathrm{b}}$, D. Reyna $^{\mathrm{b}}$, \\ B. Sur ${ }^{\mathrm{c}}$ \\ ${ }^{a}$ Lawrence Livermore National Laboratory, Livermore, CA 94550, USA \\ ${ }^{b}$ Sandia Livermore National Laboratories, Livermore, CA 94550, USA \\ ${ }^{c}$ Atomic Energy of Canada, Limited, Chalk River Laboratories, Chalk River, ON, Canada
}

\begin{abstract}
Here we present the development of a compact antineutrino detector for the purpose of nuclear reactor monitoring, improving upon a previously successful design. This paper will describe the design improvements of the detector which increases the antineutrino detection efficiency threefold over the previous effort. There are two main design improvements over previous generations of detectors for nuclear reactor monitoring: dual-ended optical readout and single volume detection mass. The dual- ended optical readout eliminates the need for fiducialization and increases the uniformity of the detector's optical response. The containment of the detection mass in a single active volume provides more target mass per detector footprint, a key design criteria for operating within a nuclear power plant. This technology could allow for real-time monitoring of the evolution of a nuclear reactor core, independent of reactor operator declarations of fuel inventories, and may be of interest to the safeguards community.

8 Keywords: nuclear reactor safeguards, antineutrino detection
\end{abstract}

\section{Introduction}

Fission reactors emit large numbers of antineutrinos and this flux may be useful for the measurement of two quantities of interest for reactor safeguards [3]: reactor power and plutonium inventory throughout an operational cycle. The high antineutrino flux produced by reactors allows relatively simple cubic meter scale detectors at tens of meters standoff to record hundreds or thousands of antineutrino events per day. Such antineutrino detectors would add online, quasi-real-time bulk material accountancy to the set of reactor monitoring tools available to safeguards agencies via a continuous, unattended and non-intrusive measurement technique.

Between 2003 and 2008, a collaboration between Lawrence Livermore National Laboratory (LLNL) and Sandia National Laboratory (SNL) successfully deployed several prototype safeguards detectors at a commercial Pressurized Light Water Reactor (PLWR) in order to test both the method and the practicality of its implementation in the field. In

\footnotetext{
${ }^{*}$ Corresponding Author. Tel.: +1 9254220268.

Email address: classen2@llnl.gov (T. Classen)

${ }^{1}$ Present Address: Candu Energy Inc., Mississauga, ON, Canada

${ }^{2}$ Present Address: Defence R\&D Canada - Centre for Security Science (CSS), Ottawa, ON, Canada 

${ }_{46} \mu \mathrm{s}$.

particular, the SONGS1 [4] detector performed power monitoring [2] and observed reactor fuel evolution [5] using the emitted $\overline{v_{e}}$ flux. The success of these and other efforts led the IAEA Novel Technologies Unit to convene an Experts Meeting in 2008 [10] and a Working Group Meeting in 2011 [11] to assess current antineutrino detection technology and examine how it might be incorporated into the safeguards regime. As noted during these discussions, detector systems with greater sensitivity than the initial SONGS1 prototype would provide more detailed information regarding reactor operation. However, maintaining simplicity and robustnesses are also important considerations, since these systems must operate unattended in the challenging environment presented by a commercial nuclear power station.

With these goals in mind, a collaboration between Lawrence Livermore National Laboratory, Sandia National Laboratory, and Atomic Energy of Canada Limited have constructed a detector designed for deployment at a nuclear power reactor. The detector incorporates significant improvements in detection efficiency, uniformity of response, and energy resolution compared with SONGS1. We anticipate that these technical improvements will make antineutrino detection technology more attractive for safeguards, thereby satisfying a key goal from the interim report [10]. Improving the sensitivity of the detector was not the sole focus of effort however, as in order for this technology to be adopted by regulators or industry, it must be demonstrated to be both affordable and convenient for operators. Consequently, the design uses off-the-shelf components where possible. Additionally, software was developed to automate the neutrino analysis, with little operator input.

Here we describe the design of the system, as well as its performance during laboratory testing. Through a combination of commissioning data and simulation we will demonstrate the system's capabilities as a compact $\bar{v}_{e}$ detector, and will present a comparison to the previous SONGS1 device.

\section{Detector Description}

\subsection{Detection Method}

The inverse beta decay reaction $\overline{v_{e}}+p \rightarrow n+e^{+}$is used to distinguish detected $\overline{v_{e}}$ 's from most natural backgrounds. The positron-neutron pair provide a coincidence signature with a time separation defined by the neutron capture time in the detection medium. Furthermore, the kinetic energy of the prompt positron is related to the $\bar{v}_{e}$ energy by the relation $E_{e^{+}} \approx E_{\overline{v_{e}}}-0.8 \mathrm{MeV}$. The neutron capture time is determined by the composition of the scintillator. In keeping with the goal of a practical demonstration, a readily available commercial scintillator was chosen. This detector contains BC-525 liquid scintillator [9], with a Gd loading of $0.1 \%$ by weight, giving a capture time of $~ 33$

\subsection{Detector Features}

The main detector volume consists of a $1.6 \mathrm{~m} \times 1.6 \mathrm{~m} \times 1.6 \mathrm{~m}$ scintillator volume, containing $\sim 3.6$ tons of liquid scintillator (Figure 1). The scintillator is contained within a reinforced stainless steel vessel, with a $2.54 \mathrm{~cm}$ air gap at the top to allow for thermal expansion and contraction of the liquid. The scintillator volume is viewed on 


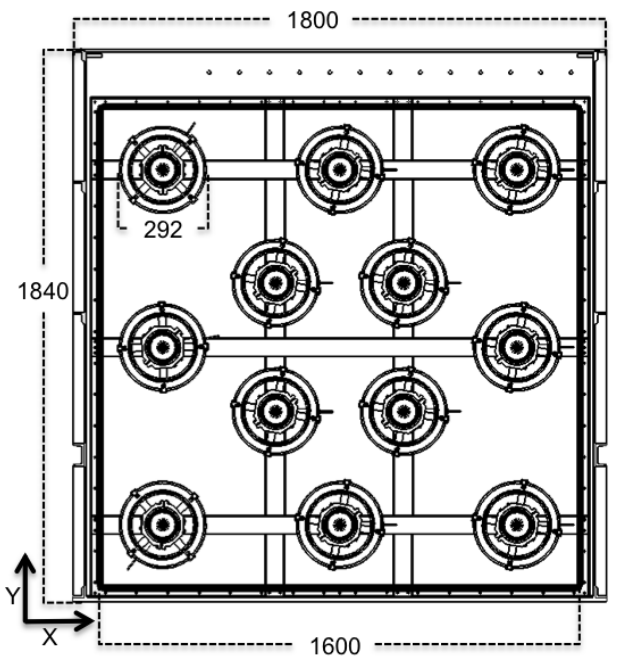
ensure the detector is isolated from external optical photons.

opposing ends through $2.54 \mathrm{~cm}$ thick acrylic windows, sealed with teflon coated Viton o-rings [8]. The dual-ended readout of this detector was a key improvement over the SONGS1 design, allowing for improved light collection uniformity, resolution and position resolution. Each window is instrumented with 12 Hamamatsu R7081 PMTs [6] (10" diameter) to detect scintillation signals. The scintillator vessel is contained within a reinforced stainless steel buffer vessel containing mineral oil to provide hydrostatic support for the acrylic windows and optical coupling of the scintillator volume to the PMTs (Figure 2A). A synthetic rubber lid is fitted across a the top of the buffer vessel to

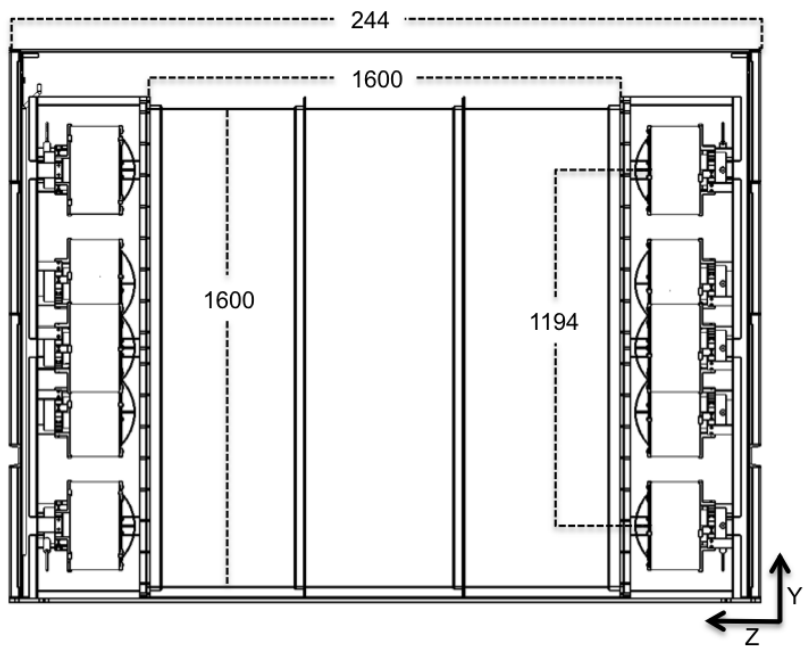

Figure 1: Two schematic views of the detector, including the inner detector and buffer vessel. All measurements are in mm.

The detector tank assembly is completely contained within a water shield to attenuate external background radiation. The water shield is composed of six interlocking stainless steel tanks providing a minimum $50 \mathrm{~cm}$ thickness of water shielding in all directions (Figure 2B). Each tank is designed such that there are no line-of-sight gaps from the exterior to the active detector volume in order to better shield the inner detector from low energy neutrons, an important source of background.

The water shield is surrounded on five sides by a muon veto system consisting of 33 plastic scintillator panels. Each panel is instrumented with two 2" PMTs to achieve more uniform light collection as a function of muon interaction position. The muon veto panels on the "north", "south" and top of the detector assembly consist of a $5 \mathrm{~cm} \times 61 \mathrm{~cm}$ x 305cm rectangular EJ200 [12] plastic scintillator panel, with triangular light guides: this is dubbed the "fish-tail" configuration. The "east" and "west" panels consist of a $5 \mathrm{~cm}$ thick rectangular scintillator panel observed by two PMTs on the outside face. Each PMT in this configuration is placed $\frac{1}{4}$ of the panel length from the end of the panel. While this configuration has worse gamma/muon discrimination than the fish-tail configuration, it allows these panels to reside within the footprint of the light guides of the 3 other sides (Figure 2C). This arrangement provides full scintillator coverage over sides excluding the bottom, and maintains a compact system footprint. The thickness of the 


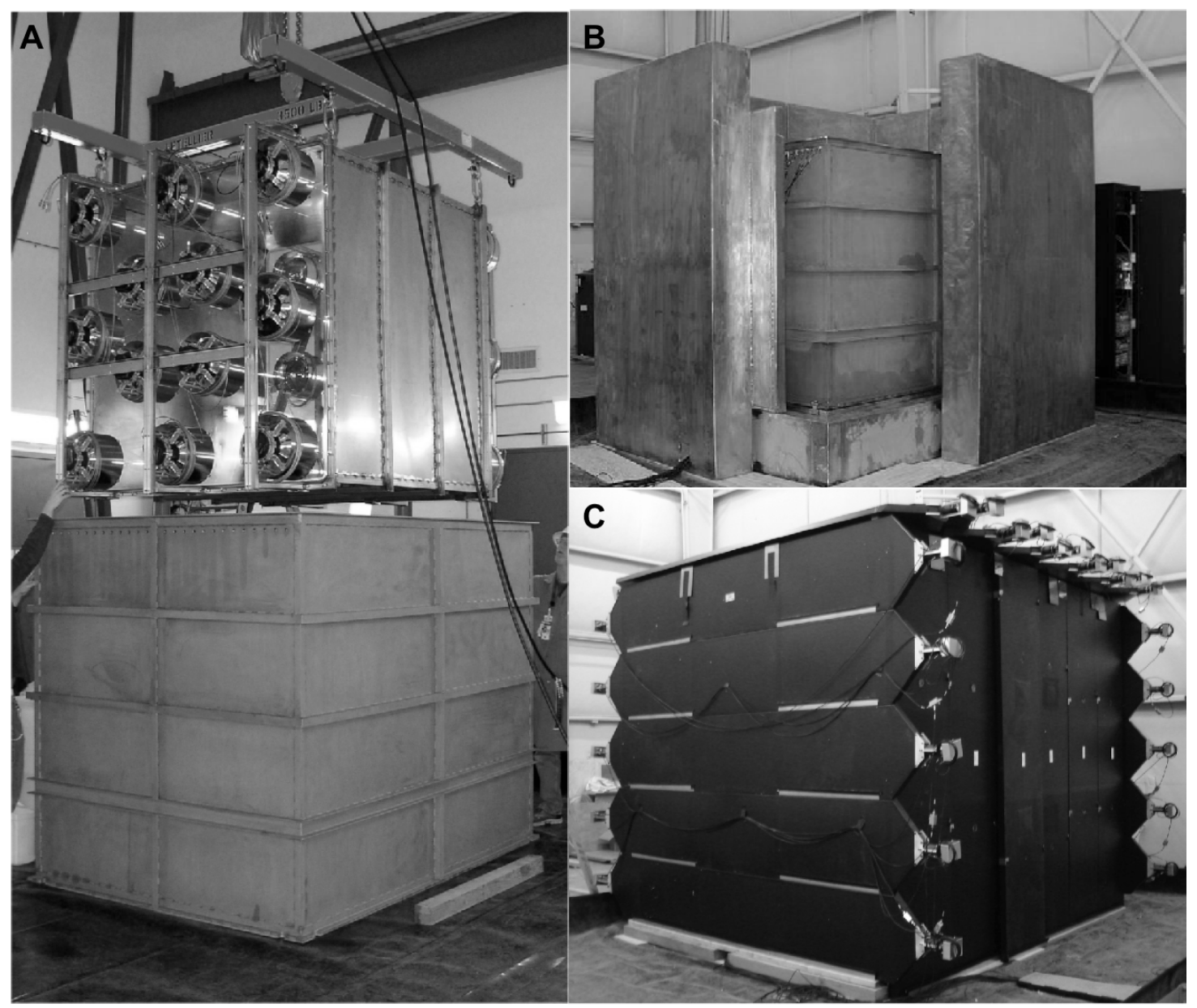

Figure 2: A) The instrumented scintillator vessel being lowered into the buffer vessel. B) The buffer vessel partially surrounded by the water shielding tanks. C) The completed detector with external muon veto system.

panels gives a clear separation between the muon signals and natural gamma background (Figure 3) to reduce muon rejection related dead time, while maintaining high muon rejection efficiency.

\subsection{Data Acquisition System}

The data acquisition system (Figure 4) is composed of flexible, commercially available components (Table 1). After high voltage decoupling, the PMT signal is passed through a low pass LC filter network to ensure that the narrow single photo-electron response covers several samples of the waveform digitizers (WFDs) used downstream. After shaping, a CAEN V795 Fast amplifier provides x10 gain and signal fan out to Struck SIS3320 WFDs (operated at $200 \mathrm{MHz}$ ) and CAEN V812 Constant Fraction Discriminators (CFDs). The Struck SIS3320 waveform digitizers were used to capture integrated PMT event charges and baselines. SIS3320 firmware that provided gated integrals was used for data reduction, two gates were defined to capture the waveform pedestal and signal. All SIS3320 WFDs are synchronized to a single Struck SIS3820 clock, and the data is transferred to the data acquisition computers by 


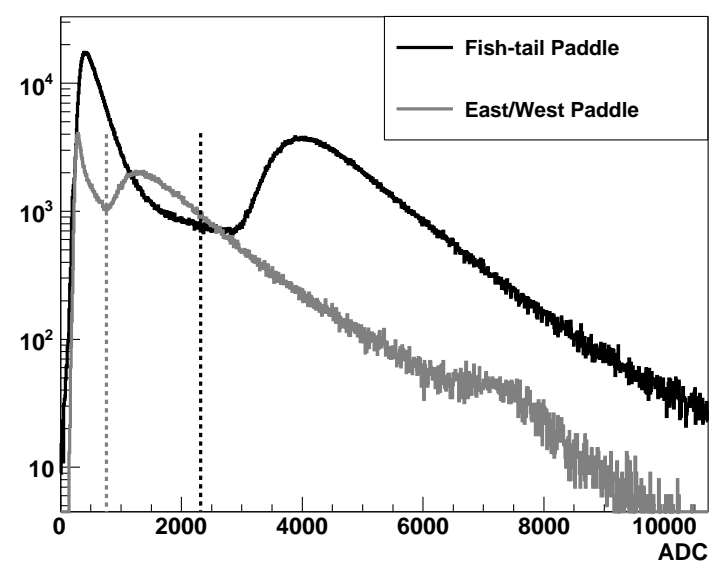

Figure 3: The ADC spectra from a "fish-tail" and "east/west" muon panel. The vertical dashed lines represent the threshold for gamma muon separation.

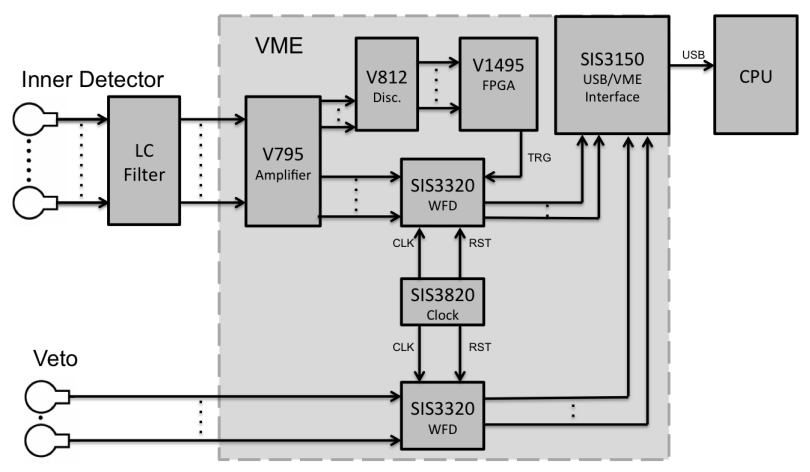

Figure 4: Schematic of the data acquisition system. 


\begin{tabular}{llll}
\hline Quantity & Item & Purpose & Link \\
\hline 1 & Struck SIS3820 & Clock & http://www.struck.de/sis3820clock.html \\
1 & Struck SIS3150 & USB / VME interface & http://www.struck.de/sis3150usb.htm \\
8 & Struck SIS3320 & ADC/Digitizer & http://www.struck.de/sis3320.htm \\
1 & CAEN V1495 & VME Board & http://www.caen.it/csite/CaenProd.jsp?parent=11\&idmod=484 \\
2 & CAEN V812 & Discriminator & http://www.caen.it/csite/CaenProd.jsp?parent=11\&idmod=46 \\
3 & CAEN V975 & Amplifier & http://www.caen.it/csite/CaenProd.jsp?idmod=254\&parent=11 \\
\hline
\end{tabular}

Table 1: Data Acquisition System

\section{Simulation}

A Geant4 [1] based simulation tool was specifically developed for this system. The simulation tool contains all detector components, and the ability to run the simulation with a complete detector, or each component individually. This allows the simulation to recreate both the fully deployed detector, as well as the various partially-constructed test phases. The simulation is able to simulate calibration sources, backgrounds, as well as the antineutrino spectra for ${ }^{235} \mathrm{U},{ }^{239} \mathrm{Pu},{ }^{238} \mathrm{U}$ and ${ }^{241} \mathrm{Pu}$. The fission isotopes can be combined with different proportions to simulate the antineutrino spectrum during all stages of a reactor cycle. The simulation performed full optical transport and applied a PMT photocathode response function to fully model the detector up to generation of photoelectrons at the photocathode. The optical reflectance and scintillation properties of the simulation materials were tuned such that the simulated response qualitatively matched the test data from the detector (Figure 5).

The PMT arrays on each end of the detector are referred to as "north" and "south". The natural log of the ratio of the north PMTs response to the south PMTs provides an estimate of the $\mathrm{Z}$ position of events within the detector (Figure 6a). This estimator breaks down near the acrylic windows where shadowing of PMTs at large angles by mu-metal shields results in a disproportionate fraction of the light produced being detected on the opposite side of the detector. The $\mathrm{Z}$ position inference is therefore biased towards that opposite end. Conversely, events occurring near the acrylic but in front of a PMT face result in that PMT receiving a disproportionately large percentage of the light, causing a misreconstruction of the inferred $\mathrm{Z}$ position beyond the actual extent of the detector.

The $\mathrm{X}$ and $\mathrm{Y}$ position of events can be inferred through a charge weighted position average along the respective axes, albeit less accurately than the $\mathrm{Z}$ position (Figure 6b). Features in these distributions are due to the mu-metal shields, and the optical limitations of the dual-ended readout. The falloff in reconstruction ability near the edges of the detector is a simple limitation of using charge sharing, as even events happening at the detector edge will have some light detected in PMTs on the opposite edge, pulling these events towards the center of the detector. The majority of the reconstruction issues are caused by the $\sim 5 \mathrm{~cm}$ proximity of the PMTs to the scintillator volume.

There are several lessons learned from studies of position reconstruction within this detector. It is preferable to leave some separation between the PMT's and the active volume to minimize reconstruction anomalies. However, this reduces the total detector volume. Additionally, PMT timing could be used to improve the position reconstruc- 

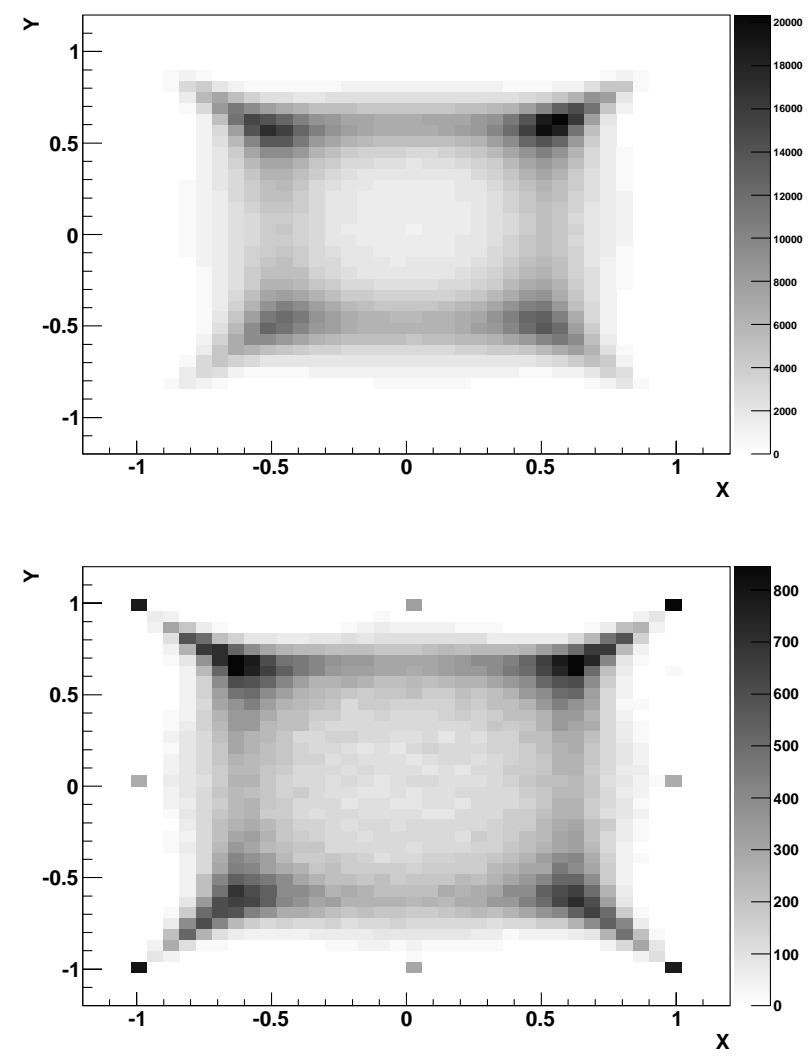

Figure 5: a) Background data reconstructed $\mathrm{Y}$ vs X positions. b) Simulation data reconstructed $\mathrm{Y}$ vs X positions. The main features, hotter corners with a uniform central region, are observed in both cases.

tion, particularly near the edges of the detector where the charge-sharing method falters. Upgrading the DAQ with waveform digitizers that operate at a higher sampling rate would allow for this type of improvement.

\section{Calibration}

\subsection{LED Calibration}

An LED is located at the center of each PMT array, which may be externally triggered in coincidence with a forced DAQ trigger. The PMT gains are then extracted from the resulting single photoelectron (SPE) spectra (Figure 7). The high voltage applied to the inner detector PMTs was tuned for identical SPE response. The DAQ software was written such that physics data would be taken in 24 hour blocks, with short LED calibration runs taken between blocks to track PMT gain stability.

\subsection{Source Calibration}

Calibrations of the inner detector were taken using ${ }^{252} \mathrm{Cf}$ and ${ }^{228} \mathrm{Th}$ (Figure 8) sources placed on the exterior of the buffer tank wall. Since the system was designed to run autonomously once installed, the design did not allow for 

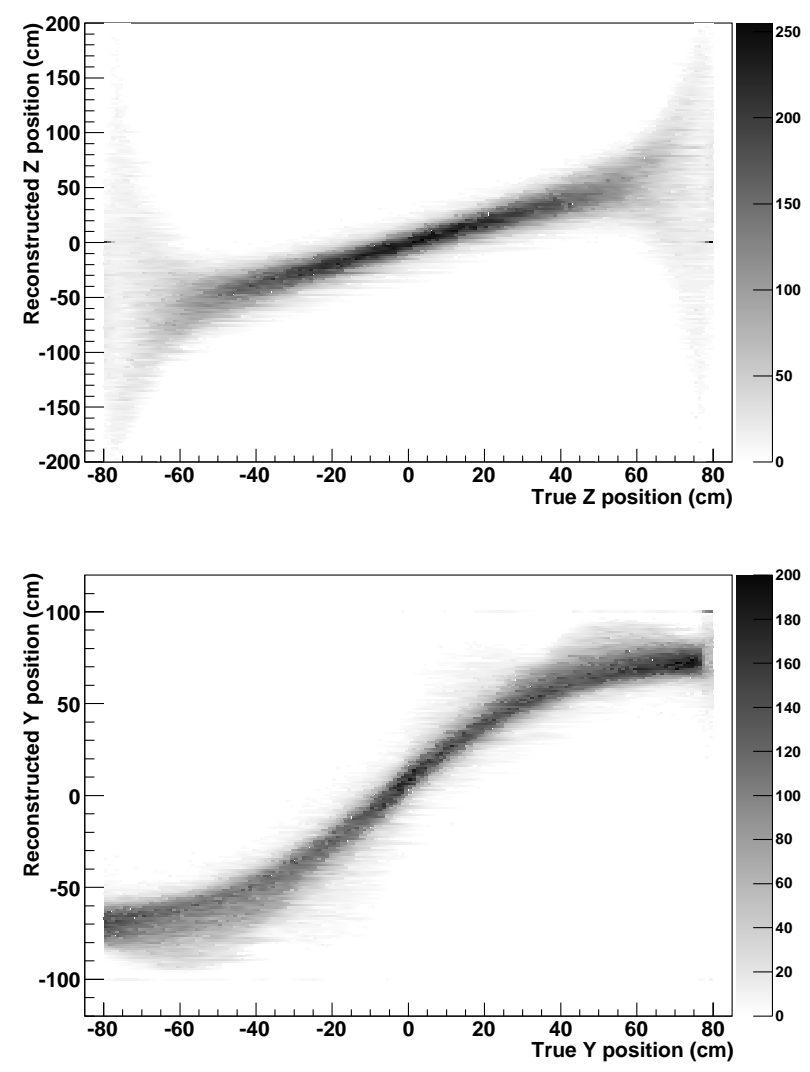

Figure 6: a) The reconstructed $\mathrm{Z}$ positions of events compared to their true positions from a Geant4 simulation. b) The reconstructed $\mathrm{Y}$ positions of events compared to their true positions from a Geant4 simulation. The $\mathrm{X}$ and $\mathrm{Y}$ dimensions are analogous in this analysis.

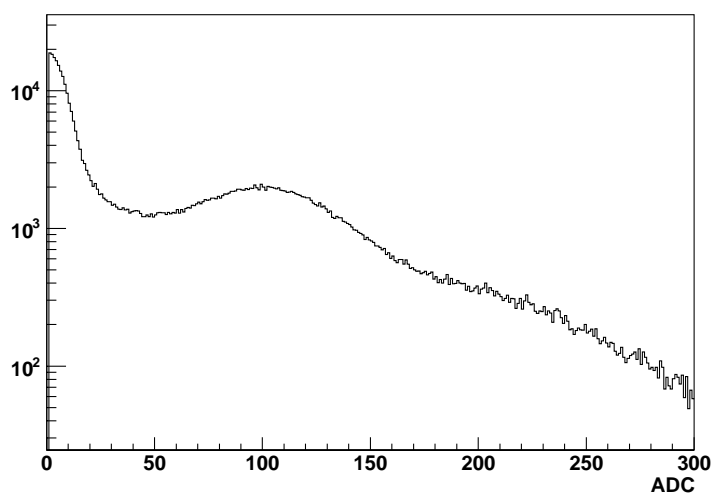

Figure 7: The Single Photoelectron(SPE) spectrum from one inner detector PMT.

internal deployment of radioactive sources. Natural background sources such as neutron capture from spallation, and natural gamma peaks would be analyzed for calibration once deployed. An automated calibration system would be a useful upgrade for future designs, but did not fit into the scope of this project. 
Analysis of the $2.6 \mathrm{MeV}$ gamma ray peak from ${ }^{228} \mathrm{Th}$, the $2.2 \mathrm{MeV}$ gamma ray peak from neutron capture on hydrogen, and the $\sim 8.5 \mathrm{MeV}$ shoulder from the neutron capture on Gd show a consistent energy resolution of $\frac{20 \%}{\sqrt{E}}$, where $\mathrm{E}$ is reconstructed energy in units of $\mathrm{MeV}$. The hydrogen and gadolinium neutron capture peaks were measured using a ${ }^{252} \mathrm{Cf}$ calibration source and spallation neutrons produced by cosmic muon interactions in the detector and its surroundings. ${ }^{252} \mathrm{Cf}$ calibration data was collected using a sealed source placed at the edge of the detector tank. A statistical background subtraction allowed the isolation of the neutron capture response [13]. A similar response spectrum for spallation neutrons was isolated by requiring events in the neutron capture energy range to occur within $100 \mu$ s of each other. The energy resolution and response of the detector is unlikely to be sufficient to perform an unfolding of the reactor antineutrino energy spectrum, allowing inference of a reactor's isotopic fission fractions. However, it could be used to verify the broad shift in spectrum expected to occur as PWR fuel evolves as in [7], and would clearly be suitable for rate-only reactor monitoring measurements of the sort demonstrated with SONGS1 [2].

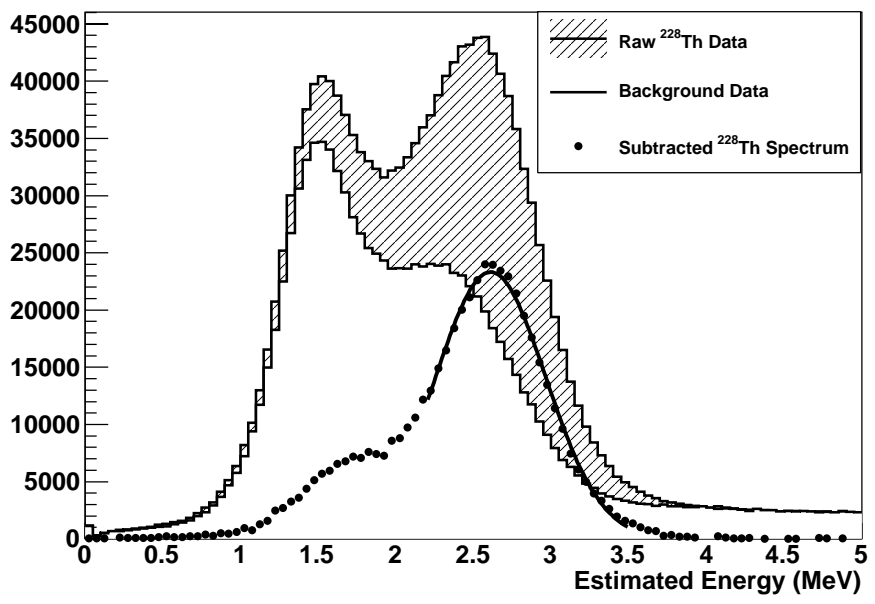

Figure 8: Fitting the ${ }^{228} \mathrm{Th}$ spectrum after background subtraction allows for calibration of the detector's energy response, as well as measurement of the detector's energy resolution.

\section{Analysis}

The dual-ended optical readout of this device allows for an improved event selection efficiency of $39.2 \%$ compared to the previous SONGS1 detector's $11.8 \%$ (Table 2). One example of this is the position reconstruction capabilities of the detector, which are evident when analyzing the calibration data. By performing background subtraction of the position distributions of source data, it becomes clear that the events are localized spatially (Figure 9).

Additionally, by taking the geometric mean of the north and south PMTs it is possible to correct for light attenuation and other propagation losses, giving a more uniform energy response. The energy resolution of the detector is improved by $15 \%$ to $\frac{20 \%}{\sqrt{E}}$ using this technique. The resolution of the detector could be improved significantly by a 

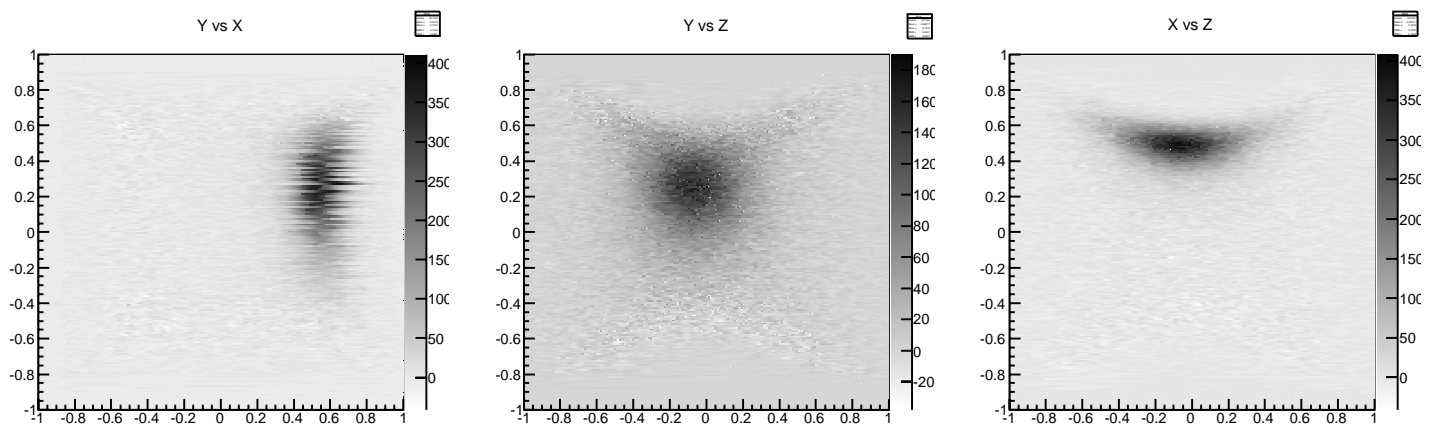

Figure 9: The background-subtracted position distributions of events from a ${ }^{228} \mathrm{Th}$ source placed on the exterior of the buffer tank. For this calibration, the source was placed on a wall in the YZ plane

detailed series of calibrations at various positions within the detector to map the position dependence of this response, although such an effort was not completed here. The position dependence of the energy response was further studied by examining the simulated response to uniformly distributed mono-energetic positrons (Figure 10). The resolution of a central region containing $12 \%$ of the active volume, compared to the entire active volume, is a factor of two better in this example, although some part of that gain is due to the improved likelihood of capturing the positron's annihilation gamma's in the central region.

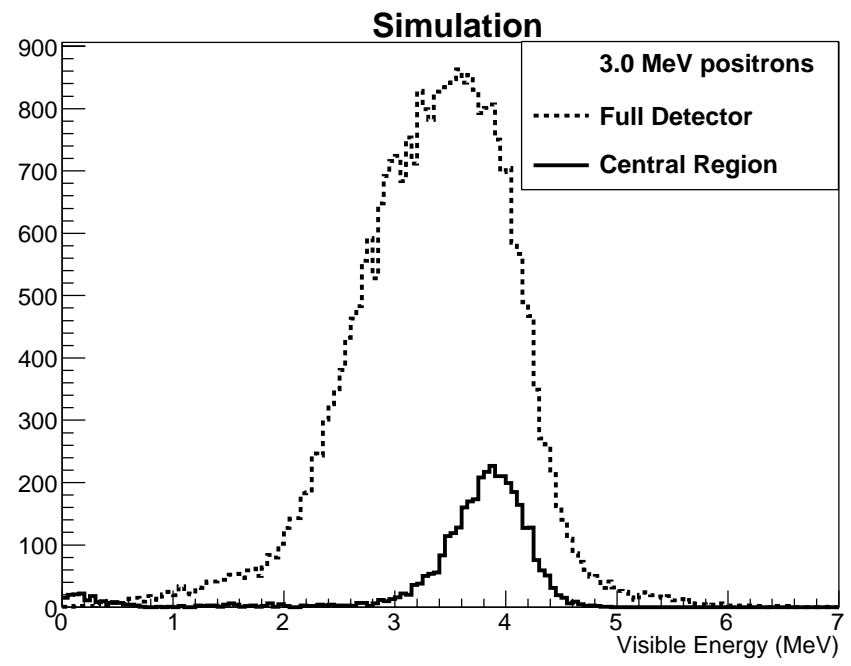

Figure 10: The reconstructed energy spectrum for simulated 3.0 MeV positrons in the whole detector and a central region, including annihilation gamma rays.

The detector has no particle identification capabilities, e.g. via pulse shape discrimination, so selection of antineutrino interactions relies on measuring the time between, and energy of, events. By examining the interval between events in the neutron capture energy range, it is possible to simultaneously fit the neutron capture time constant and 
accidental coincidence rates [4]. Spallation neutrons were used to measure the neutron capture time in the scintillator, as they provide an abundant correlated neutron capture signal at the Earth's surface. A fit to the inter-event time distribution (Figure 11) of background data gives a neutron capture time of $33 \mu \mathrm{s}$, and an accidental coincidence rate consistent with the uncorrelated background.

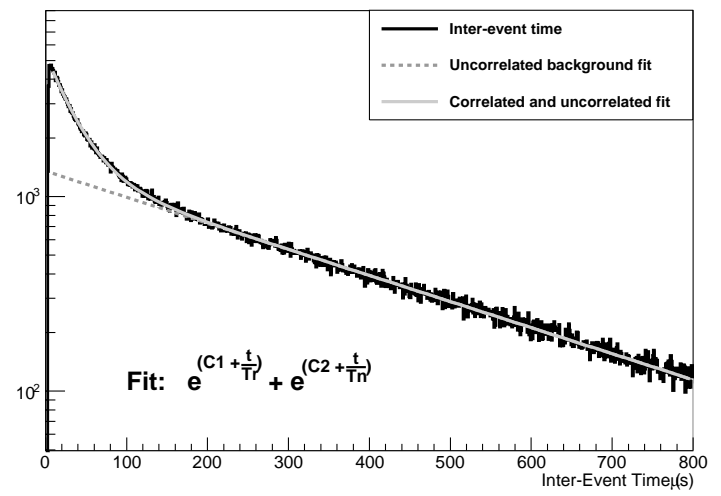

Figure 11: Inter-event time for background events.

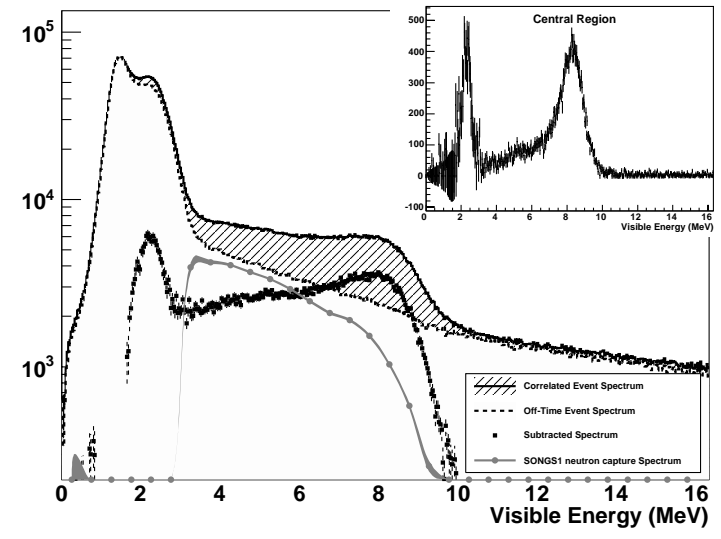

Figure 12: Neutron capture energy response from multiple neutron capture due to spallation. The difference between the energy response at the short (solid black) and long (dashed black) inter-event times is due to the neutron capture response (hatched). The difference of these yields the neutron capture response(blue). For comparison, the SONGS neutron capture response is also shown (red). The inset shows this detectors neutron capture spectrum within a central detector region, illustrating full capture of the Gd neutron capture cascade

To demonstrate the ability of this instrument as a $\bar{v}_{e}$ detector, we estimate the efficiency of the various components of the total $\overline{v_{e}}$ detection efficiency and perform a comparison with that obtained during the previous SONGS1 demonstration (Table 2). Some components, such as the muon veto efficiency, were not calculable, as they depend heavily on the specific background and signal rates at a deployment site, so these values were not considered here. 


\subsection{Positron Efficiency}

The positron detection efficiency is most strongly affected by the analysis threshold applied to the first event in a correlated pair. For the sake of comparison we assume the same threshold as used in the SONGS1 demonstration of $2.39 \mathrm{MeV}$. In fact it may be possible to lower this threshold, and therefore increase this efficiency, if it could be shown that additional cuts, such as inter-event distance (Figure 13) prove to be effective against random coincidence backgrounds. However such analyses must be made after deployment, when the actual background conditions are known. Therefore, in the comparison performed here, the only area in which this detector bests SONGS1 is a higher percentage of captured annihilation gamma rays. The gains however are meager, giving a positron detection efficiency of $60 \pm 6 \%$.

In order to estimate the positron efficiency, as well as its error, we must estimate the error on the energy scale of the detector. This was done by first calculating the effect on the positron energy reconstruction of ignoring scintillator quenching when performing the gamma energy calibration. The non-linearity of the gamma ray calibration points available was calculated for a range of quenching parameters reasonable for scintillators of this type. The quenched parameters were linearly fit to extract a calibration constant, which was applied to the positron energy scale. The difference between the linearly calibrated positron energies and positron energies with the quenching function applied was calculated, and the maximum difference of $4 \%$ was taken as the non-linearity component of the energy scale error. This value was added in quadrature to the fit error on the gamma calibration data for a total energy scale error of $5 \%$.

The positron spectrum obtained from the beta decay of fission fragments for a reactor core at equilibrium was simulated to estimate the positron efficiency. The positron efficiency error was estimated by simulating one million random thresholds from a Gaussian distribution peaked at $2.39 \mathrm{MeV}$, with a width of $5 \%(0.12 \mathrm{MeV})$. The integral of the reactor $\bar{v}_{e}$ positron energy spectrum was calculated above these thresholds with the results tabulated in a histogram. This histogram was fit with a Gaussian distribution, with the centroid of the distribution at $60 \%$ giving the positron efficiency, and the width of $5 \%$ giving the error on the efficiency.

\subsection{Neutron Efficiency}

Though there is a small gain in positron efficiency, significant gains were achieved in neutron detection efficiency. A spectrum taken from the accidental only portion of the inter-event time range can be subtracted from a spectrum in the neutron capture range to extract the neutron capture energy spectrum (Figure 12). The accidental time range is calculated so as to contain an equal number of accidental background coincidences as the time interval in the neutron capture signal region. The Gd capture full energy shoulder $(8.5 \mathrm{MeV})$ and neutron capture on hydrogen $(2.2 \mathrm{MeV})$ are clearly visible. The Gd shoulder is more distinct in this detector than in SONGS1, and the hydrogen capture peak is clearly visible. For a direct comparison to the SONGS1 detector, setting the neutron capture threshold at the SONGS1 value of $3.5 \mathrm{MeV}$ gives an efficiency of $69 \%$. A conservative error of $5 \%$ is obtained by shifting the neutron capture cut by the energy resolution of the detector and observing the change in efficiency. Thus the detector maintains excellent neutron identification efficiency above a significant portion of the natural gamma ray background. The inset 
of Figure 12 shows the neutron capture energy spectrum, with a position cut restricting events to the central portion of the detector. Though the position reconstruction is uncalibrated, this clearly demonstrates that full containment of the Gd neutron capture gamma ray shower is occurring in the detector for a subset of events, driving the major improvement to the neutron detection efficiency over SONGS1.

\subsection{DAQ LiveTime}

The SIS3320 WFDs are configured with a dual bank event buffer, so there is no dead time associated with data readout of the system at the $\sim 1 \mathrm{kHz}$ event rates encountered during commissioning and expected during system deployments. A $2 \mu$ s trigger delay between events results in the only DAQ dead time. The unshielded background rate with no overburden in the laboratory test area was $3000 \mathrm{~Hz}$, resulting in a DAQ dead time of $0.6 \%$. Once the shield was added,the background trigger rate was reduced to $50 \%$ of the unshielded value, for a maximum trigger dead time of $0.3 \%$. The trigger dead time is highly dependent on the specific deployment site characteristics, but the laboratory test area represents a worst case scenario, and the dead time at any site is expected to be below the unshielded location with no overburden. This yields a minimum trigger efficiency of $99.7 \pm 0.3 \%$.

\subsection{Minimum $\Delta t$}

The neutron capture timing efficiency was calculated by integrating over the sum of two exponential functions $e^{-t / 33.3}-e^{-t / 1.5}$ where $33 \mu \mathrm{s}$ is the measured neutron capture time, and $\mathrm{e}^{1.5 \mu s}$ is an approximate representation of the neutron thermalization time that matches the shape in the measured Gd capture timing data at low inter-event time. The efficiency was calculated by integrating this formula from 2 to $100 \mu \mathrm{s}$, and dividing by the total integral of the function to give a value of $95 \%$. The error on this value was estimated by evaluating the same formula with neutron capture times altered by the measured neutron capture time error yielding $0.9 \%$.

\subsection{Fiducial Volume}

The dual ended readout of the detector ensures that all events generated within the detector are visible to at least half of the PMTs. Thus the full fiducial volume may be used, in contrast to the SONGS1 detector. The ability to calculate an inter-event position metric however (Figure 13) means that an additional data cut could be used instead of a fiducial volume. Since the inverse-beta decay positron and neutron are produced in the same position, they will be detected with a small inter-event distance. Accidental background however will have inter-event distances spanning all distances allowed by the detector. Analysis of simulation data shows that cutting on this parameter would cut the accidental background rate in half, while maintaining $99 \%$ efficiency for detectable inverse beta decay events. A more aggressive cut would remove $75 \%$ of accidental backgrounds while maintaining a $95 \%$ efficiency for detectable inverse beta decay events. These cuts are ineffective for natural correlated backgrounds. As stated above, any decision to use such an analysis must be informed by the actual site background spectrum. 


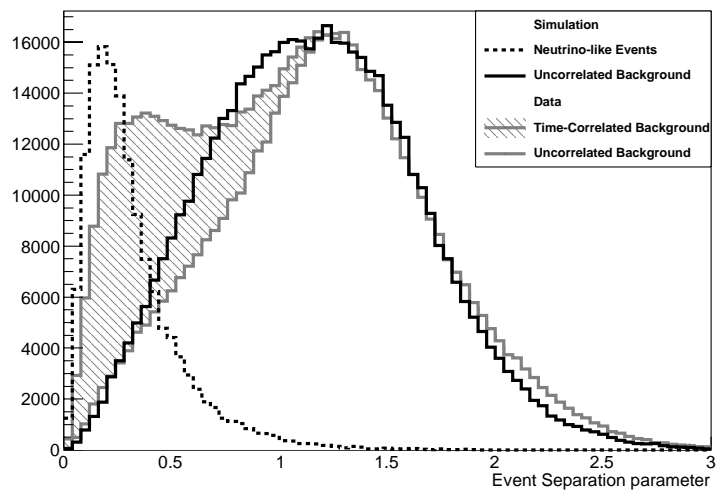

Figure 13: The event separation metric for simulation and data. Both simulation and data show the random coincidence distribution that peaks at larger separation. Additionally the event separation for events in the correlated data sample shown in Figure 11 (a mixture of correlated signals and random coincidences) and simulation of inverse-beta decay events are shown.

\subsection{Total Efficiency}

A simple Monte Carlo simulation was used to estimate the total detector efficiency and error from the individual components. Each component was represented in the simulation as a Gaussian distribution peaking at the component efficiency, with a width of the component efficiency error. Random values were chosen from each Gaussian distribution for one million fake experiments, and multiplied to calculate each experiments total efficiency. The total efficiencies were recorded in a histogram, which fit well to a Gaussian distribution. The mean of this fit was taken as the total detector efficiency, and the width was taken as the error on the total efficiency. The combination of the many improvements made in this detector design result in a total detection efficiency three times greater than SONGS1.

\begin{tabular}{ccc}
\hline Quantity & SONGS1 Efficiency & Current Detector Efficiency \\
\hline Positron & $55 \pm 5 \%$ & $60 \pm 6 \%$ \\
Neutron & $40 \pm 4 \%$ & $69 \pm 5 \%$ \\
DAQ Live Time & $92 \pm 0.5 \%$ & $99.7 \pm 0.3 \%$ \\
Minimum $\Delta \mathrm{t}$ & $70 \pm 0.5 \%$ & $95 \pm 0.9 \%$ \\
Fiducial & $83 \pm 4 \%$ & $100 \%$ \\
\hline Total & $11.8 \pm 1.6 \%$ & $39.2 \pm 4.7 \%$ \\
\hline$\frac{\text { target mass }}{\text { footprint }}$ & $0.11 \frac{\text { ton }}{\mathrm{m}^{2}}$ & $0.36 \frac{\text { ton }}{\mathrm{m}^{2}}$ \\
$\frac{\text { Detections }}{\text { Day }}$ at SONGS & 400 & 7500 \\
Table 2: Detection efficiency comparison between SONGS1 and the system described here.
\end{tabular}


The deployed footprint of this detector, including the water shield and muon veto system, is $10 \mathrm{~m}^{2}$, compared to $6 \mathrm{~m}^{2}$ for the SONGS1 device. Within this footprint, the current design contains 3.6 tons of target mass with a detection efficiency of $39.2 \%$, whereas the SONGS1 device obtained only 0.64 tons of target mass at $11.8 \%$ detection efficiency. If the two systems were deployed in the same location at a reactor complex, the current design would observe a factor of 18.7 more $\bar{v}_{e}$. Alternately, this detector could be deployed at 4.3 times the distance from the reactor core as SONGS1, and have identical performance, significantly increasing the likelihood of finding a suitable deployment position within an existing power plant.

A simulation was developed to generate expected datasets for this detector in the original SONGS1 deployment location during a reactor shut down. For this simulation, a conservative signal to background ratio of $\frac{1}{3}$ was used as a worst-case extrapolation of the SONGS1 background. Additionally, the neutrino flux decayed with a 15 minute time constant after shutdown to simulate continued beta decay within the fuel elements. Applying the sequential probability ratio test as in [2], the reactor shutdown is detected within 90 minutes for $99.5 \%$ of 1 million simulated data sets, with a mean detection time of 49 minutes. This compares favorably to the 300 minute detection time from SONGS1, though it must be stressed that this analysis depends heavily on assumed background conditions, whereas the SONGS1 analysis used actual data.

\section{Conclusion}

An advanced antineutrino detector for nuclear reactor monitoring has been designed and constructed. The detector design features more detection mass per footprint, dual-ended readout, and enhanced shielding/muon rejection compared to previous systems. An antineutrino detection efficiency of $39.2 \%$ has been estimated for this system from extensive testing. Additional improvements for background shielding and rejection have been tested, including a modular water shield, and a thick plastic muon rejection system. The significant improvements to the detector efficiency result in improved deployability, as well as reactor monitoring ability. The detection system is complete, and currently available for deployment, if a suitable reactor site can be found.

\section{Acknowledgements}

This work was supported by the U.S. Department of Energy Office of Defense Nuclear Nonproliferation Research and Development.

This work was performed under the auspices of the U.S. Department of Energy by Lawrence Livermore National Laboratory under Contract DE-AC5-07NA27344. LLNL-JRNL-655279.

Sandia National Laboratories is a multi-program laboratory managed and operated by Sandia Corporation, a wholly owned subsidiary of Lockheed Martin Corporation, for the U.S. Department of Energys National Nuclear Security Administration under contract DE-AC04-94AL85000. SAND2014-15109O. 


\section{References}

[1] Agostinelli, S., Allison, J., Amako, K., Apostolakis, J., Araujo, H., Arce, P., Asai, M., Axen, D., Banerjee, S., Barrand, G., Behner, F., Bellagamba, L., Boudreau, J., Broglia, L., Brunengo, A., Burkhardt, H., Chauvie, S., Chuma, J., Chytracek, R., Cooperman, G., Cosmo, G., Degtyarenko, P., Dell'Acqua, A., Depaola, G., Dietrich, D., Enami, R., Feliciello, A., Ferguson, C., Fesefeldt, H., Folger, G., Foppiano, F., Forti, A., Garelli, S., Giani, S., Giannitrapani, R., Gibin, D., Cadenas, J. G., Gonzlez, I., Abril, G. G., Greeniaus, G., Greiner, W., Grichine, V., Grossheim, A., Guatelli, S., Gumplinger, P., Hamatsu, R., Hashimoto, K., Hasui, H., Heikkinen, A., Howard, A., Ivanchenko, V., Johnson, A., Jones, F., Kallenbach, J., Kanaya, N., Kawabata, M., Kawabata, Y., Kawaguti, M., Kelner, S., Kent, P., Kimura, A., Kodama, T., Kokoulin, R., Kossov, M., Kurashige, H., Lamanna, E., Lampn, T., Lara, V., Lefebure, V., Lei, F., Liendl, M., Lockman, W., Longo, F., Magni, S., Maire, M., Medernach, E., Minamimoto, K., de Freitas, P. M., Morita, Y., Murakami, K., Nagamatu, M., Nartallo, R., Nieminen, P., Nishimura, T., Ohtsubo, K., Okamura, M., O’Neale, S., Oohata, Y., Paech, K., Perl, J., Pfeiffer, A., Pia, M., Ranjard, F., Rybin, A., Sadilov, S., Salvo, E. D., Santin, G., Sasaki, T., Savvas, N., Sawada, Y., Scherer, S., Sei, S., Sirotenko, V., Smith, D., Starkov, N., Stoecker, H., Sulkimo, J., Takahata, M., Tanaka, S., Tcherniaev, E., Tehrani, E. S., Tropeano, M., Truscott, P., Uno, H., Urban, L., Urban, P., Verderi, M., Walkden, A., Wander, W., Weber, H., Wellisch, J., Wenaus, T., Williams, D., Wright, D., Yamada, T., Yoshida, H., Zschiesche, D., 2003. Geant4?a simulation toolkit. Nuclear Instruments and Methods in Physics Research Section A: Accelerators, Spectrometers, Detectors and Associated Equipment 506 (3), 250 - 303.

URL http://www.sciencedirect.com/science/article/pii/S0168900203013688

[2] Bernstein, A., Bowden, N. S., Misner, A., Palmer, T., 2008. Monitoring the thermal power of nuclear reactors with a prototype cubic meter antineutrino detector. Journal of Applied Physics 103 (7).

URL http://scitation.aip.org/content/aip/journal/jap/103/7/10.1063/1.2899178

[3] Bernstein, A., Wang, Y., Gratta, G., West, T., 2002. Nuclear reactor safeguards and monitoring with antineutrino detectors. Journal of Applied Physics 91 (7), 4672-4676.

URL http://scitation. aip.org/content/aip/journal/jap/91/7/10.1063/1.1452775

[4] Bowden, N., Bernstein, A., Allen, M., Brennan, J., Cunningham, M., Estrada, J., Greaves, C., Hagmann, C., Lund, J., Mengesha, W., Weinbeck, T., Winant, C., 2007. Experimental results from an antineutrino detector for cooperative monitoring of nuclear reactors. Nuclear Instruments and Methods in Physics Research Section A: Accelerators, Spectrometers, Detectors and Associated Equipment 572 (2), 985 998.

URL http://www.sciencedirect.com/science/article/pii/S0168900206024326

[5] Bowden, N. S., Bernstein, A., Dazeley, S., Svoboda, R., Misner, A., Palmer, T., 2009. Observation of the isotopic evolution of pressurized water reactor fuel using an antineutrino detector. Journal of Applied Physics 105 (6).

URL http://scitation.aip.org/content/aip/journal/jap/105/6/10.1063/1.3080251

[6] catalog, H. P. D., 6 2014. http://www.hamamatsu.com/resources/pdf/etd/p-dev_ 2014_toth0022e01.pdf.

[7] Christensen, E., Huber, P., Jaffke, P., Shea, T., 2014. Antineutrino monitoring for the Iranian heavy water reactor. Phys.Rev.Lett. 113, 042503.

[8] ENCAP-O-SEAL, 6 2014. http://www.m-cor.com/.

[9] Piepke, A. G., Moser, S., Novikov, V. M., 1999. Development of a gd loaded liquid scintillator for electron anti-neutrino spectroscopy. Nuclear Instruments and Methods in Physics Research Section A: Accelerators, Spectrometers, Detectors and Associated Equipment 432 (2?3), 392 -398 .

URL http://www.sciencedirect.com/science/article/pii/S0168900299005306

[10] Report, I., 10 2008. Final report: Focused workshop on antineutrino detection for safeguards applications.

[11] Report, I., 2011. Proceedings of the first meeting of the ad hoc working group on safeguards applications utilizing antineutrino detection and monitoring, sG-EQ-GNRL-RP-0002.

[12] scintillator data sheet, E. E.-. P., 6 2014. http://www.eljentechnology.com/index.php/component/content/article/31-general/48-ej-200.

[13] Sweany, M., Bernstein, A., Bowden, N., Dazeley, S., Keefer, G., Svoboda, R., Tripathi, M., 2011. Large-scale gadolinium-doped water cherenkov detector for nonproliferation. Nuclear Instruments and Methods in Physics Research Section A: Accelerators, Spectrometers, 
Detectors and Associated Equipment 654 (1), 377 - 382.

URL http://www.sciencedirect.com/science/article/pii/S016890021101165X 